\title{
Long Josephson Junction Stack Coupled to a Cavity
}

\section{Madsen, Søren Peder; Pedersen, Niels Falsig; Groenbech-Jensen, N.}

Published in:

IEEE Transactions on Applied Superconductivity

Link to article, DOI:

10.1109/TASC.2007.898049

Publication date:

2007

Document Version

Publisher's PDF, also known as Version of record

Link back to DTU Orbit

Citation (APA):

Madsen, S. P., Pedersen, N. F., \& Groenbech-Jensen, N. (2007). Long Josephson Junction Stack Coupled to a Cavity. IEEE Transactions on Applied Superconductivity, 17(2), 926. https://doi.org/10.1109/TASC.2007.898049

\section{General rights}

Copyright and moral rights for the publications made accessible in the public portal are retained by the authors and/or other copyright owners and it is a condition of accessing publications that users recognise and abide by the legal requirements associated with these rights.

- Users may download and print one copy of any publication from the public portal for the purpose of private study or research.

- You may not further distribute the material or use it for any profit-making activity or commercial gain

- You may freely distribute the URL identifying the publication in the public portal

If you believe that this document breaches copyright please contact us providing details, and we will remove access to the work immediately and investigate your claim 


\title{
Long Josephson Junction Stack Coupled to a Cavity
}

\author{
S. Madsen, N. F. Pedersen, and N. Grønbech-Jensen
}

\begin{abstract}
A stack of inductively coupled long Josephson junctions are modeled as a system of coupled sine-Gordon equations. One boundary of the stack is coupled electrically to a resonant cavity. With one fluxon in each Josephson junction, the inter-junction fluxon forces are repulsive. We look at a possible transition, induced by the cavity, to a bunched state.
\end{abstract}

Index Terms-Cavity, fluxons, THz oscillator.

\section{INTRODUCTION}

$\mathbf{R}$ ESEARCH on stacks of long Josephson junctions have been on-going for many years [1]-[3]. The system is nonlinear in nature and therefore hard to understand completely but at the same time very interesting. Fluxon solutions are large amplitude non-linear electromagnetic excitations, which can be perturbed by several external factors such as an applied bias current or an external magnetic field [4]. One possible application for the long Josephson junction stack is generation of radiation in the hundreds of $\mathrm{GHz}$ range. The fluxons can be made to shuttle back and forth in the stack and will radiate when near the edges of the stack. The radiation from a single junction is very small and very hard, but possible, to detect [5]. For applications the radiated power must be increased and one way to obtain this is to have all the individual junctions in the stack radiate coherently. However, fluxons in different junctions repel each other, preventing the formation of a coherent state. In Ref. [6] it was shown for weakly coupled junctions, that an applied microwave field may induce coherence. The method had some drawbacks for applications, such as the need to apply microwaves. In this paper we look at an alternative, but highly related, scheme. We couple the junctions to a cavity which will act as the external microwave source of Ref. [6], and through the cavity coherence will be induced for weakly coupled systems.

Section II introduces the model and shows how we model the coupling to an external cavity. Section III explains our numerical procedure and conducts the numerical experiments. This is the main part, and shows that it is possible for the cavity to overcome the fluxon-fluxon repulsion for stacks with different parameters. Section IV concludes the paper.

Manuscript received August 27, 2006. Supported by the Danish STVF program "New Superconductors" and by the UC Davis Center for Digital Security under the AFOSR Grant FA9550-04-1-0171.

S. Madsen and N. F. Pedersen are with Oersted-DTU, Section of Electric Power Engineering, The Technical University of Denmark, DK-2800 Kgs. Lyngby, Denmark.

N. Grønbech-Jensen is with the Department of Applied Science, University of California, Davis, CA 95616 USA.

Digital Object Identifier 10.1109/TASC.2007.898049

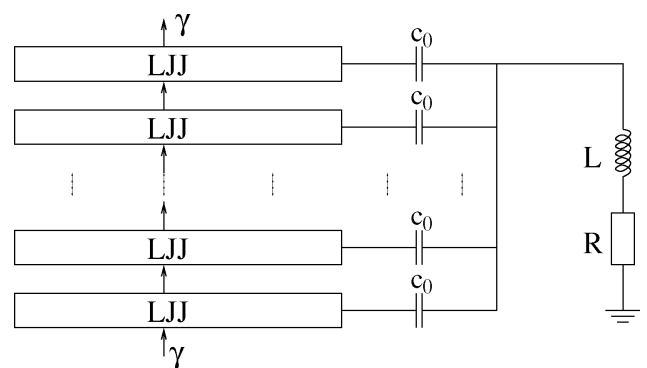

Fig. 1. The model. $N$ inductively coupled long Josephson junctions with an applied bias current, $\gamma$, are coupled to a cavity. The cavity is modeled by a $R L C$ circuit.

\section{MODEL}

To model a stack of identical long Josephson junctions with $N+1$ superconducting layers and $N$ insulating layers we use [7]

$$
\mathbf{J}=\mathbf{S}^{-1} \boldsymbol{\phi}_{x x}
$$

where the $i$ th element of $\phi, \phi^{i}$, is the gauge invariant phase difference across insulating layer $i$. The $N \times N$ coupling matrix, $\mathbf{S}$, is given by

$$
\mathbf{S}=\left(\begin{array}{ccccc}
1 & S & & & \\
S & 1 & S & & \\
& S & 1 & S & \\
& & \ddots & \ddots & \ddots
\end{array}\right)
$$

with $S$ being the coupling parameter between the layers [7]. The vector $\mathbf{J}$ has the components

$$
J^{i}=\phi_{t t}^{i}+\alpha \phi_{t}^{i}+\sin \phi^{i}-\gamma,
$$

where the $\alpha$-term is a dissipative term, and $\gamma$ is the bias-current in the z-direction.

Equations (1)-(3) are written in normalized units. Space, $x$, is normalized to the Josephson penetration depth, $\lambda_{J}=\sqrt{\hbar / 2 e \mu_{0} J^{c} d^{\prime}}$, and time, $t$, is normalized to the inverse plasma frequency, $\omega_{0}^{-1}=\sqrt{\hbar c_{J} / 2 e J^{c}}$, where the symbols have their usual meaning, see Ref. [7] for details.

The model of the Josephson stack coupled to a series cavity is shown in Fig. 1. The boundary conditions for the phases can be written as [8]

$$
\begin{aligned}
\phi_{x}^{i}(0, t) & =0 \text { and } \\
\phi_{x}^{i}(L, t) & =\frac{\dot{q}}{N}-\frac{c}{N^{2}} \sum_{k=1}^{N}\left(\phi_{t t}^{k}(L, t)-\phi_{t t}^{i}(L, t)\right),
\end{aligned}
$$

where $c=N c_{0} / c_{J}$ is the normalized capacitance and $q$ is the normalized charge in the cavity. Defining $\Omega=1 /\left(\omega_{c} \sqrt{N L c_{0}}\right)$ 
to be the normalized cavity frequency and $Q=\sqrt{L /\left(N R^{2} c_{0}\right)}$ as the quality factor, the linear cavity equation becomes

$$
\frac{d^{2} q}{d t^{2}}+\frac{\Omega}{Q} \frac{d q}{d t}+\Omega^{2} q=\Omega^{2} \frac{c}{N} \sum_{i=1}^{N} \phi_{t}^{i}(L, t) .
$$

For more details on these equations see Ref. [8].

Two terms are present in (5). The first term couples the junctions to the cavity, by equally dividing the cavity current between the $N$ junctions. The second term represents a direct coupling between the junction through the capacitors $c_{0}$. Since the junctions would in an experiment be embedded in a resonator and couple through electro-magnetic radiation from the edges, this second term models the radiation leaving one junction and ending up in another junction without being reflected by the cavity. With an efficient cavity, the second term is therefore expected to be much smaller than the first term and may safely be neglected. We thus choose to consider

$$
\phi_{x}^{i}(0, t)=0 \quad \text { and } \quad \phi_{x}^{i}(L, t)=\frac{\dot{q}}{N}
$$

as the boundary conditions for (1)-(3) and (6). Numerical calculations with both type of boundary conditions show that the second term in (5) indeed has little effect on the overall behavior of the system, even in the model situation shown in Fig. 1 where the effect of the second term is expected to be much greater than in a real experiment.

\section{NumericAl CALCULATIONS}

To solve the non-linear equations (1)-(3) with boundary conditions (6) and (7) we use 2 nd order finite differences for the spatial derivatives and a 5th order Runge-Kutta method for the time-development. The numerical simulations are done by varying (decreasing and subsequent increasing) the applied bias current, keeping all other parameters fixed. This enables us to change the fluxon shuttling frequency and trace out the current-voltage characteristic of the system. Since there are many parameters involved, we choose to settle on $L=4, \alpha=0.1$, and $Q=100$ in all our simulations. We start out at high bias current and then lower it gradually. Each simulation is run for a time long enough to (possibly) stabilize the cavity current. When a simulation is ended the amplitude and frequency of the cavity current, the fluxon shuttling frequency, the fluxon-fluxon phases and the fluxon-cavity current phases are calculated. The phases and frequencies are calculated by making a Fourier transform of $\phi^{i}(L, t)$ and then using the frequency and phase with maximum power. A new simulation is thereafter started with a smaller bias current and the output of the previous simulation as initial conditions. Sometimes we found the need to first decrease the bias current gradually and then increase it gradually in order to trace out more of the current-voltage characteristic. To determine the frequencies and phases, we use linear interpolation of the time evolution data and a fast Fourier transform.

Shown in Fig. 2 is a simulation for three junctions with $S=$ $-0.01, \Omega=0.7$, and $c=0.004$. The top plot shows the current-voltage characteristic of the system, as the bias current is scanned, first from high to low and then from low to high. Hysteretic behavior of the system is seen, and the maximum value
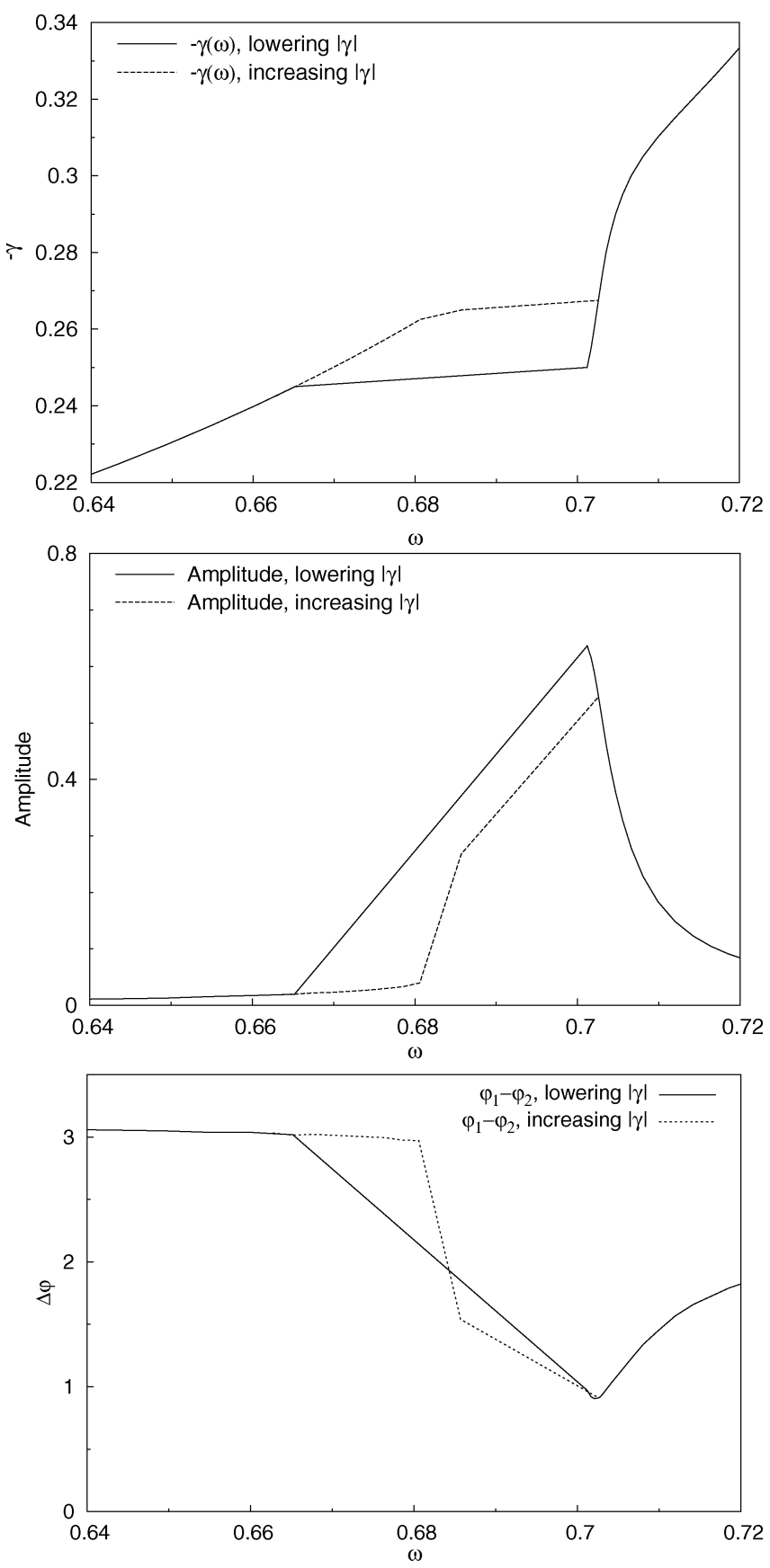

Fig. 2. Numerical simulation with $N=3, L=4, \alpha=0.1, S=-0.01$, $\Omega=0.7, Q=100$, and $c=0.004$. (Top) Current-Fluxon frequency (voltage) characteristic. (Middle) Amplitude of the cavity current. (Bottom) Phase difference. The cavity pushes the fluxons together, but not into a bunched state.

of the perturbation from no-cavity is found when scanning from high to low. The maximum perturbation is found for $\omega=\Omega$, where $\omega$ is the fluxon shuttling frequency (voltage).

The middle plot in Fig. 2 shows the amplitude of the cavity current $\dot{q}$ as a function of the fluxon shuttling frequency. The cavity current exhibits a narrow peak with maximum at $\omega=\Omega$. It is seen that when scanning from high to low, the maximum of the resonance is encountered, before the system switches to a lower voltage. When scanning from low to high, the center of the resonance is not encountered. This is in agreement with the current voltage characteristic. 
The lower plot shows the phase-difference between the fluxons in junctions number 1 and 3 and the fluxon in junction in number 2 (fluxons in junction number 1 and 3 have the exact same phase due to symmetry). When going from high to low frequency, the phase difference is seen to decrease, and reach a minimum when $\omega \approx \Omega$. The cavity current has the effect of acting like an oscillating magnetic field, which has been shown to be able to press two repulsive fluxons into a bunched state [6].

In Fig. 2 the cavity did not succeed in bunching the fluxons as the minimum of the phase difference between the fluxons was not zero or near zero. The cavity did, however, increase the coherence. In Fig. 3 a calculation is shown where the cavity does succeed in creating an in-phase bunched state from an initial anti-phase moving state. The difference between the systems in Figs. 2 and 3 is that we have lowered the cavity frequency, $\Omega$, and increased the cavity-junction coupling, $c$. The latter has the effect of increasing the cavity current, as more energy is drained from the junctions into the cavity, resulting in a larger cavity current. The effect of decreasing the cavity frequency is, that the maximum value of the cavity current is now when the fluxon shuttling frequency is $\omega=0.6$, resulting in a lower average fluxon velocity. The repulsive force between the fluxons is known to depend heavily on the fluxon velocity [10], so the decreasing of the cavity frequency has the effect of decreasing the fluxon repulsion at resonance.

When decreasing the cavity frequency, $\Omega$, one also decreases the absolute value of the bias current at resonance. The value of the coupling, $c$, also has this effect. When the bias current is small, there may not be enough energy left to restore the fluxons after a collision with the boundary [9]. Thus, the cavity frequency, $\Omega$, and coupling strength, $c$, should be chosen such that one is able to come close to the resonance frequency, while still preserving the fluxons in the system. This fact has the unfortunate effect, that one can not readily choose a very low cavity frequency in order to keep the fluxon-fluxon repulsion at a minimum near resonance. In Fig. 3 there is a sudden drop in cavity current amplitude at $\omega \approx 0.615$ corresponding to $\gamma \approx-0.18$. This drop is due to the fact, that the middle fluxon have been annihilated, and the junction is therefore not releasing much energy into the cavity and the amplitude drops. In the phase-difference plot, this is seen as a large increase between the phase-difference between the signals in junctions 1 and 2 .

The most interesting effect in Fig. 3 is shown in the bottom plot. As the fluxon frequency gets closer to the cavity frequency, the cavity current amplitude increases. This has the effect of pushing the repulsive fluxons together, and for a small range of $\omega$ it is seen that the phase difference between junction 1 and 2 reaches a finite minimum just above zero. In this range, the fluxons are bunched. For the case of two junctions, this minimum would have been zero phase difference, but due to the asymmetry of the three-junction case, there is a small residual phase [11].

Fig. 4 shows the same type of calculations as Figs. 2 and 3 but instead with 6 junctions. As the resonance frequency is approached, the relative phases of the fluxons are lowered. At $\omega \approx 0.615$, the system is essentially in a bunched state, which exists until $\omega \approx 0.61$. Here, the fluxons in junctions 2 and 5 are
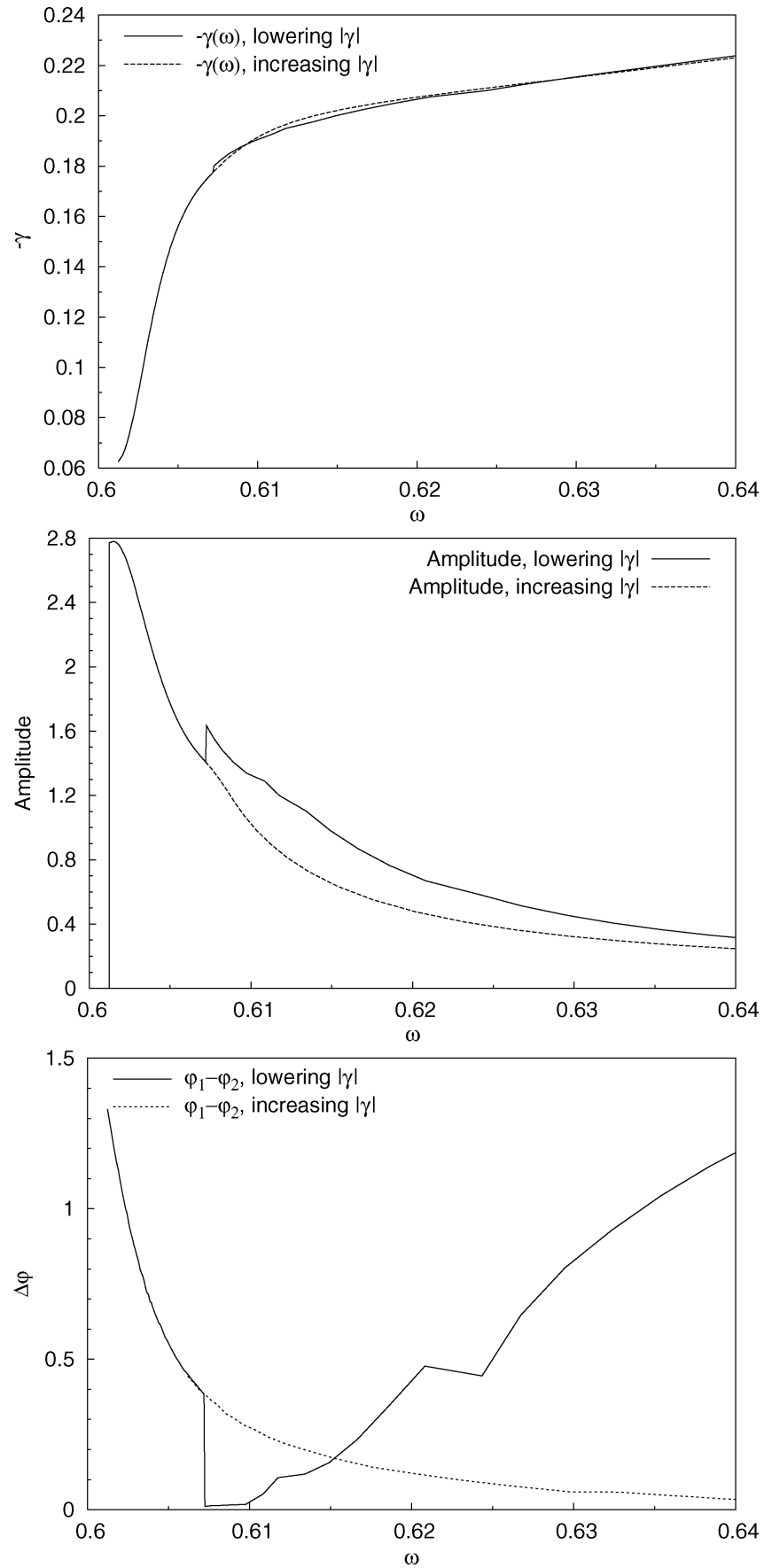

Fig. 3. Numerical simulation with $N=3, L=4, \alpha=0.1, S=-0.01$, $\Omega=0.6, Q=100$, and $c=0.04$. (Top) Current-Fluxon frequency (voltage) characteristic. (Middle) Amplitude of the cavity current. (Bottom) Phase difference. The cavity pushes the fluxons together, and near resonance the fluxons are in a bunched state.

annihilated, while the fluxons in junctions $1,3,4$, and 6 remain in the bunched state. There is a corresponding lowering of the cavity current amplitude, when the fluxons are annihilated. The current-fluxon frequency also show a small jump, and it should be noted that the relation to the current-voltage characteristic is changed below $\omega=0.61$, since the voltage of the system is proportional to the number of fluxons in the system which is lowered at this point. 

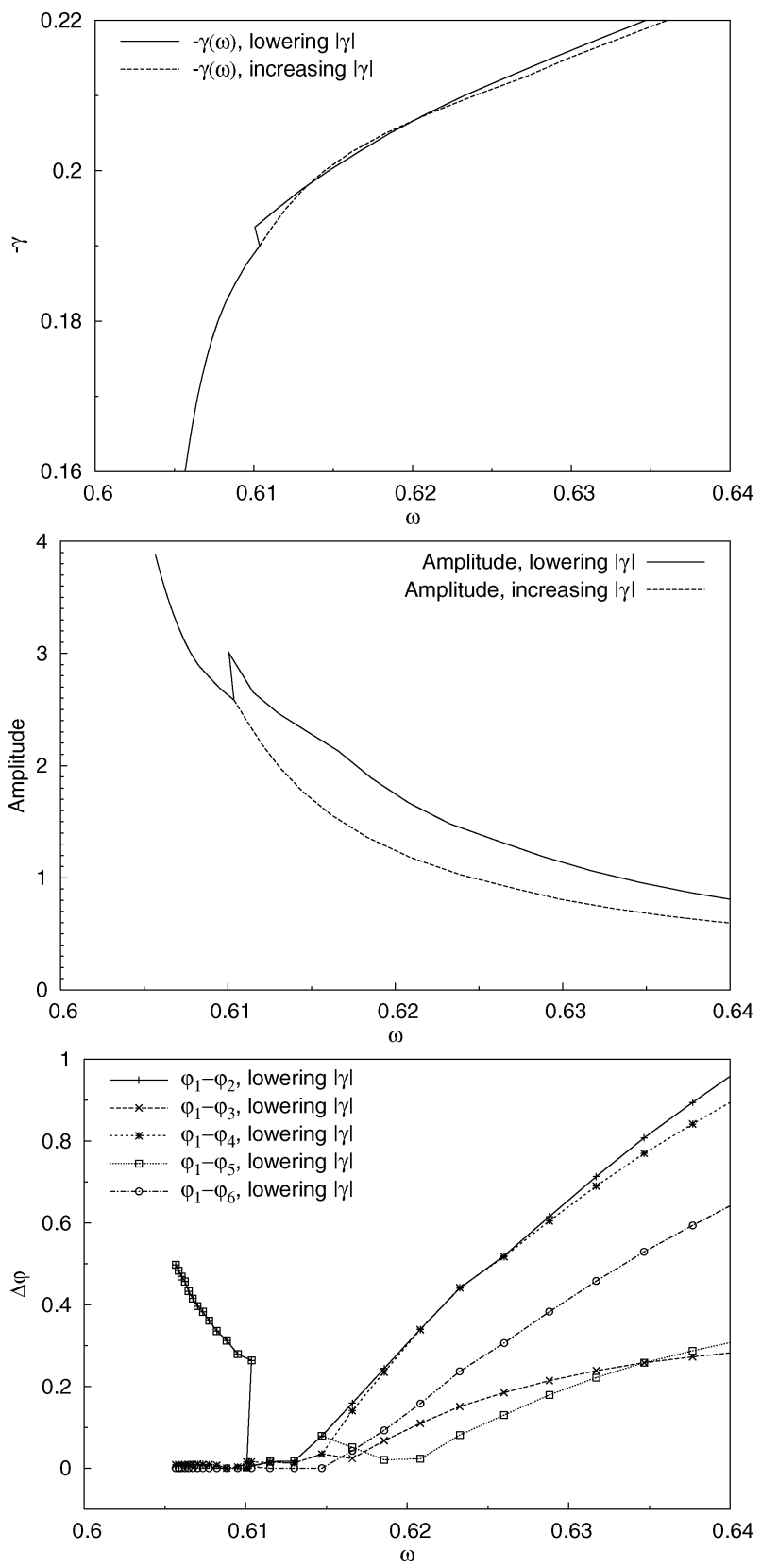

Fig. 4. Numerical simulation with $N=6, L=4, \alpha=0.1, S=-0.01, \Omega=$ $0.6, Q=100$, and $c=0.1$. (Top) Current-Fluxon frequency (voltage) characteristic. (Middle) Amplitude of the cavity current. (Bottom) Phase difference.

\section{CONCLUSION}

We have considered a system of stacked junction coupled to a high $Q$ cavity. Through numerical simulations, we have shown that it is possible for the cavity to turn an anti-phase moving fluxon configuration into an in-phase moving fluxon configuration, i.e. induce bunching. The effect is, however, rather small and can really only be used for weakly coupled junctions. The bunching effect may be enhanced by choosing a small cavity resonance frequency, small junction length, and high coupling to the cavity. However, all these parameters should be chosen such that the fluxons survive a collision with the junction edge.

\section{REFERENCES}

[1] R. Kleiner, F. Steinmeyer, G. Kunkel, and P. Müller, "Intrinsic Josephson effects in $\mathrm{Bi} 2 \mathrm{Sr} 2 \mathrm{CaCu} 2 \mathrm{O} 8$ single crystals," Phys. Rev. Lett., vol. 68, p. 2394, 1992.

[2] H. B. Wang, P. H. Wu, and T. Yamashita, "Terahertz responses of intrinsic Josephson junctions in high TC superconductors," Phys. Rev. Lett., vol. 87, p. 107002, 2001.

[3] A. A. Yurgens, "Intrinsic Josephson junctions: Recent developments," Supercond. Sci. Technol, vol. 13, p. R85, 2000

[4] D. W. McLaughlin and A. C. Scott, "Perturbation analysis of fluxon dynamics," Phys. Rev. A, vol. 18, p. 1652, 1978.

[5] A. Ustinov, private communication.

[6] S. Madsen and N. Grønbech-Jensen, "Microwave induced fluxon bunching in weakly coupled Josephson junctions," Phys. Rev. B., vol. 71, p. 132506, 2005.

[7] S. Sakai, P. Bodin, and N. F. Pedersen, "Fluxons in thin film superconductor-insulator superlattices," J. Appl. Phys., vol. 73, p. 2411, 1993.

[8] S. Madsen, G. Filatrella, and N. F. Pedersen, "Interaction between a BSCCO-type intrinsic Josephson junction and a microwave cavity," Euro. Phys. J. B, vol. 40, no. 2, p. 209, 2004.

[9] N. F. Pedersen, M. R. Samuelsen, and D. Welner, "Soliton annihilation in the perturbed sine-Gordon system," Phys. Rev. B, vol. 30, p. 4057 , 1984.

[10] N. Grønbech-Jensen, M. R. Samuelsen, P. S. Lomdahl, and J. A. Blackburn, "Bunched soliton states in weakly coupled sine-Gordon systems," Phys. Rev. B, vol. 42, p. 3976, 1990.

[11] C. Gorria, P. L. Christiansen, Y. B. Gaididei, V. Muto, N. F. Pedersen, and M. P. Sørensen, "Fluxons and their interactions in a system of three stacked Josephson junctions," Phys. Rev. B, vol. 68, p. 035415 , 2003. 